\title{
Microcontroller Based Home Automation System With Security
}

\author{
Inderpreet Kaur (Asstt. Prof.) \\ Rayat and Bahra Institute of Engineering and Bio-technology, Mohali, India \\ Email: inder_preet74@yahoo.com
}

\begin{abstract}
With advancement of technology things are becoming simpler and easier for us. Automatic systems are being preferred over manual system. This unit talks about the basic definitions needed to understand the Project better and further defines the technical criteria to be implemented as a part of this project.
\end{abstract}

Keywords-component; Automation, 8051 microcontroller, LDR, LED, ADC, Relays, LCD display, Sensors, Stepper motor

\section{INTRODUCTION}

With advancement of technology things are becoming simpler and easier for us. Automation is the use of control systems and information technologies to reduce the need for human work in the production of goods and services. In the scope of industrialization, automation is a step beyond mechanization. Whereas mechanization provided human operators with machinery to assist them with the muscular requirements of work, automation greatly decreases the need for human sensory and mental requirements as well. Automation plays an increasingly important role in the world economy and in daily experience.

Automatic systems are being preferred over manual system. Through this project we have tried to show automatic control of a house as a result of which power is saved to some extent.

\section{HOME AUTOMATION}

Home/office automation is the control of any or all electrical devices in our home or office, whether we are there or away. Home/office automation is one of the most exciting developments in technology for the home that has come along in decades. There are hundreds of products available today that allow us control over the devices automatically, either by remote control; or even by voice command.

Home automation (also called domotics) is the residential extension of "building automation". It is automation of the home, housework or household activity. Home automation may include centralized control of lighting, HVAC (heating, ventilation and air conditioning), appliances, and other systems, to provide improved convenience, comfort, energy efficiency and security. Disabled can provide increased quality of life for persons who might otherwise require caregivers or institutional care.
A home automation system integrates electrical devices in a house with each other. The techniques employed in home automation include those in building automation as well as the control of domestic activities, such as home entertainment systems, houseplant and yard watering, pet feeding, changing the ambiance "scenes" for different events (such as dinners or parties), and the use of domestic robots. Devices may be connected through a computer network to allow control by a personal computer, and may allow remote access from the internet.

Typically, a new home is outfitted for home automation during construction, due to the accessibility of the walls, outlets, and storage rooms, and the ability to make design changes specifically to accommodate certain technologies. Wireless systems are commonly installed when outfitting a preexisting house, as they reduce wiring changes. These communicate through the existing power wiring, radio, or infrared signals with a central controller. Network sockets may be installed in every room like AC power receptacles.

Although automated homes of the future have been staple exhibits for World's Fairs and popular backgrounds in science fiction, complexity, competition between vendors, multiple incompatible standards and the resulting expense have limited the penetration of home automation to homes of the wealthy or ambitious hobbyists.

\section{NEED OF AUTOMATION}

Earlier, we looked into the face of future when we talked about automated devices, which could do anything on instigation of a controller, but today it has become a reality.

a) An automated device can replace good amount of human working force, moreover humans are more prone to errors and in intensive conditions the probability of error increases whereas, an automated device can work with diligence, versatility and with almost zero error.

Replacing human operators in tasks that involve hard physical or monotonous work.

Replacing humans in tasks done in dangerous environments (i.e. fire, space, volcanoes, nuclear facilities, underwater, etc)

Performing tasks that are beyond human capabilities of size, weight, speed, endurance, etc. 
$\square \quad$ Economy improvement. Automation may improve in economy of enterprises, society or most of humankind. For example, when an enterprise that has invested in automation technology recovers its investment, or when a state or country increases its income due to automation like Germany or Japan in the 20th Century.

b) This is why this project looks into construction and implementation of a system involving hardware to control a variety of electrical and electronics system.

\section{SUPPY UNIT}

Initial stage of every electronic circuit is power supply system which provides required power to drive the whole system. The specification of power supply depends on the power requirement and this requirement is determined by its rating. The main components used in supply system are:

$\begin{array}{ll}> & \text { transformer } \\ > & \text { rectifier } \\ > & \text { input filter } \\ > & \text { regulator } \\ > & \text { output filter } \\ > & \text { output indication }\end{array}$

\section{A. Transformer:}

The main source of power(Fig 1) supply is a transformer. The maximum output power of power supply is dependent on maximum output power of transformer. We determine power from its current and voltage rating. e.g.: if there is a transformer of $12 \mathrm{~V}, 500 \mathrm{~mA}$ then maximum power delivered by transformer is $6 \mathrm{Watt}$.

It means we can drive a load from this transformer up to $6 \mathrm{w}$. In our project our maximum power requirement is $1 \mathrm{watt}$. So to provide this power we use $12 \mathrm{~V} / 250 \mathrm{~mA}$ transformer. The maximum output power of this transformer is 4 watt.it means it can easily drive load up to 4 watt.

\section{B. Rectifier}

Rectifier is a circuit which is used to convert ac to dc. Every electronic circuit requires a dc power supply for rectification. We have used four diodes.

\section{Input filter:}

After rectification we obtain dc supply from ac but it is not pure dc it may have some ac ripples .To reduce these ripples we use filters. It comprises of two filters -low frequency ripple filter and high frequency ripple filter.

To reduce low frequency ripples we use electrolytic capacitor. The voltage rating of capacitor must be double from incoming dc supply. It blocks dc and passes ripples to ground.

\section{Regulator:}

Regulator is a device which provides constant output voltage with varying input voltage. There are two types of regulators-

(a) Fixed voltage regulator

(b) Adjustable regulator

We have used fixed voltage regulator LM78XX last two digits signify output voltage. The voltage for our system is $5 \mathrm{~V}$ that is why we have used 7805 regulator which provides $5 \mathrm{~V}$ from $12 \mathrm{~V}$ dc.

\section{E. Output filter:}

It is used to filter out output ripple if any.

\section{F. Output indication}

We use LED to observe the functioning of our system. If the LED glows it confirms proper functioning of our supply. We have used four power supply units.

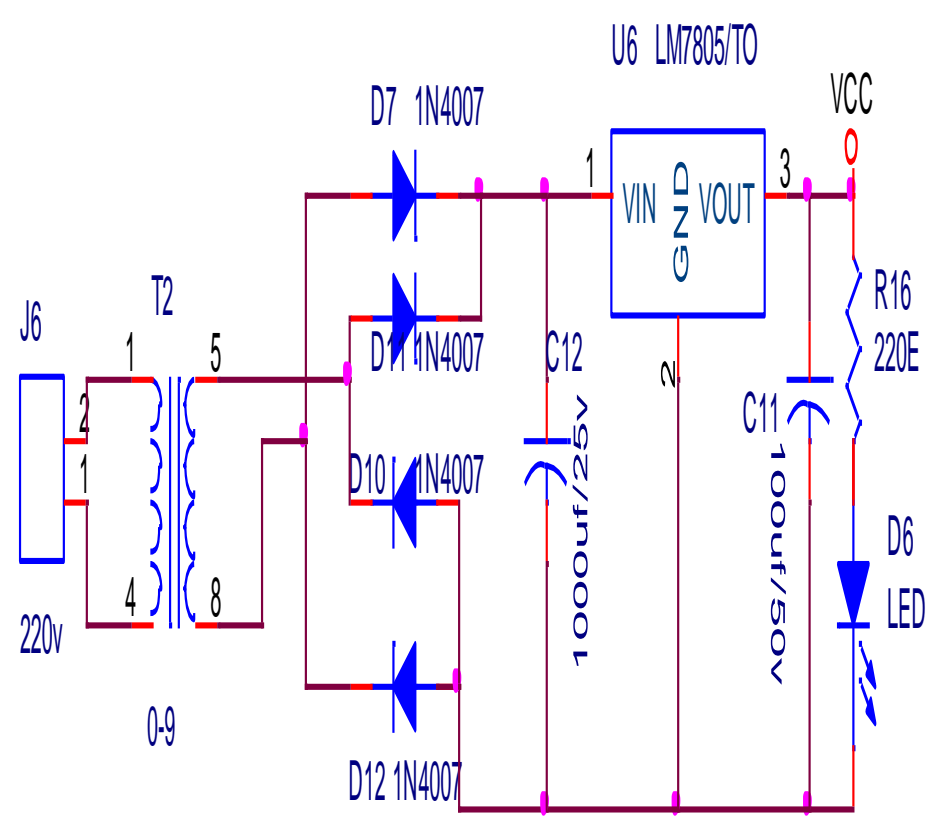

Fig 1-volt DC supply

This supply is for the microcontroller, display and relay unit. . The microcontroller requires 5 volt supply to perform any desired task.

\section{G. Control Unit}

Two control units were used one for internal system and one for external system and these control unit based on ATMEL'sAT89S52 microcontroller(Fig 2). The given capture shows the pins and basic requirement of microcontroller to make it functional. Detailed description of the controller is 
given

as

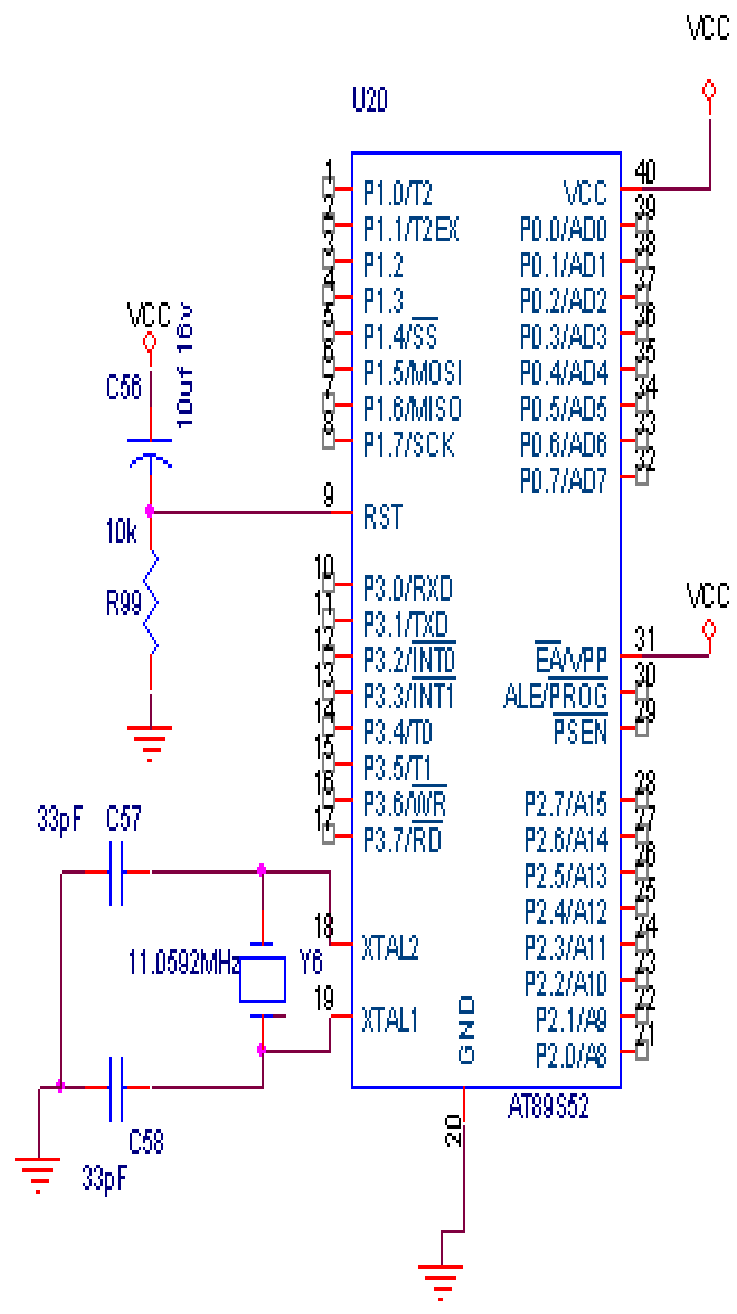

Fig 2-Chip Board

AT89S52 is an ATMEL controller with the core of Intel MCS-51. It has same pin configuration as give above. The AT89S52 is a low-power, high-performance CMOS 8-bit microcomputer with $8 \mathrm{~K}$ bytes of Downloadable Flash programmable and erasable read only memory and $2 \mathrm{~K}$ bytes of EEPROM. The device is manufactured using Atmel's high density nonvolatile memory technology and is compatible with the industry standard 80C51 instruction set and pin out.

The on-chip Downloadable Flash allows the program memory to be reprogrammed in-system through an SPI serial interface or by a conventional nonvolatile memory programmer. By combining a versatile 8-bit CPU with Downloadable Flash on a monolithic chip, the Atmel AT89S52 is a powerful microcomputer which provides a highly flexible and cost effective solution to many embedded control applications.

The AT89S52 provides the following standard features: $8 \mathrm{~K}$ bytes of Downloadable Flash, 2K bytes of EEPROM, 256 bytes of RAM, $32 \mathrm{I} / \mathrm{O}$ lines, programmable watchdog timer, two Data Pointers, three 16-bit timer/counters, a six-vector two-level interrupt, a full duplex serial port, on-chip oscillator, and clock circuitry.

In addition, the AT89S52 is designed with static logic for operation down to zero frequency and supports two software selectable power saving modes. The Idle Mode stops the CPU while allowing the RAM, timer/counters, serial port, and interrupt system to continue functioning. The Power down Mode saves the RAM contents but freezes the oscillator, disabling all other chip functions until the next interrupt or hardware reset.

The Downloadable Flash can be changed a single byte at a time and is accessible through the SPI serial interface. Holding RESET active forces the SPI bus into a serial programming interface and allows the program memory to be written to or read from unless Lock Bit 2 has been activated.

\section{H. Features \\ $>$ Compatible with MCS-51 $1{ }^{\mathrm{TM} P r o d u c t s}$ \\ $>8 \mathrm{~K}$ bytes of In-System Reprogrammable Downloadable Flash Memory \\ $>$ SPI Serial Interface for Program Downloading \\ $>$ Endurance: 1,000 Write/Erase Cycles \\ > $4.0 \mathrm{~V}$ to $5.5 \mathrm{~V}$ Operating Range \\ > Fully Static Operation: $0 \mathrm{~Hz}$ to $33 \mathrm{MHz}$ \\ $>256 \times 8$ bit Internal RAM \\ $>32$ Programmable I/O Lines \\ $>$ Three 16 bit Timer/Counters \\ $>$ Eight Interrupt Sources \\ $>\quad$ Full Duplex UART Serial Channel \\ $>$ Low Power Idle and Power Down Modes \\ $>$ Interrupt Recovery from Power Down Mode \\ I. Advantages \\ $>$ Less power consumption \\ $>$ Low cost \\ $>$ Less space required \\ $>$ High speed}

\section{J. Pin Description}

VCC: Supply voltage. GND: Ground., Port 0: Port 0 is an 8-bit open drain bidirectional I/O port. As an output port, each pin can sink eight TTL inputs. When $1 \mathrm{~s}$ are written to port 0 pins, the pins can be used as high impedance inputs. Port 0 can also be configured to be the multiplexed low-order address/data bus during accesses to external program and data memory. In this mode, P0 has internal pull-ups. Port 0 also receives the code bytes during Flash programming and outputs the code bytes during program verification. External pull-ups are required during program verification. 
Port 1: Port 1 is an 8-bit bidirectional I/O port with internal pull-ups. The Port 1 output buffers can sink/source four TTL inputs. When $1 \mathrm{~s}$ are written to Port 1 pins, they are pulled high by the internal pull-ups and can be used as inputs. As inputs, Port 1 pins that are externally being pulled low will source current (IIL) because of the internal pull-ups.

In addition, P1.0 and P1.1 can be configured to be the timer/counter 2 external count input (P1.0/T2) and the timer/counter 2 trigger input (P1.1/T2EX), respectively, as shown in the following table. Port 1 also receives the low-order address bytes during Flash programming and verification.

Port 2: Port 2 is an 8-bit bidirectional I/O port with internal pull-ups. The Port 2 output buffers can sink/source four TTL inputs. When 1s are written to Port 2 pins, they are pulled high by the internal pull-ups and can be used as inputs. As inputs, Port 2 pins that are externally being pulled low will source current (IIL) because of the internal pull-ups.

Port 2 emits the high-order address byte during fetches from external program memory and during accesses to external data memory that uses 16-bit addresses (MOVX @ DPTR). In this application, Port 2 uses strong internal pull-ups when emitting 1s. During accesses to external data memory that uses 8-bit addresses (MOVX @ RI), Port 2 emits the contents of the P2 Special Function Register. Port 2 also receives the highorder address bits and some control signals during Flash programming and verification.

Port 3 Port 3 is an 8-bit bidirectional I/O port with internal pull-ups. The Port 3 output buffers can sink/source four TTL inputs. When 1s are written to Port 3 pins, they are pulled high by the internal pull-ups and can be used as inputs. As inputs, Port 3 pins that are externally being pulled low will source current (IIL) because of the pull-ups.

Port 3 receives some control signals for Flash programming and verification. Port 3 also serves the functions of various special features of the AT89S52.

RST: Reset input. A high on this pin for two machine cycles while the oscillator is running resets the device. This pin drives high for 98 oscillator periods after the Watchdog times out. The DISRTO bit in SFR AUXR (address 8EH) can be used to disable this feature. In the default state of bit DISRTO, the RESET HIGH out feature is enabled.

ALE/PROG: Address Latch Enable (ALE) is an output pulse for latching the low byte of the address during accesses to external memory. This pin is also the program pulse input (PROG) during Flash programming. In normal operation, ALE is emitted at a constant rate of $1 / 6$ the oscillator frequency and may be used for external timing or clocking purposes. Note, however, that one ALE pulse is skipped during each access to external data memory.

If desired, ALE operation can be disabled by setting bit 0 of SFR location 8EH. With the bit set, ALE is active only during a MOVX or MOVC instruction. Otherwise, the pin is weakly pulled high. Setting the ALE-disable bit has no effect if the microcontroller is in external execution mode.
PSEN: Program Store Enable (PSEN) is the read strobe to external program memory. When the AT89S52 is executing code from external program memory, PSEN is activated twice each machine cycle, except that two PSEN activations are skipped during each access to external data memory.

EA/VPP: External Access Enable. EA must be strapped to GND in order to enable the device to fetch code from external program memory locations starting at $0000 \mathrm{H}$ up to FFFFH. Note, however, that if lock bit 1 is programmed, EA will be internally latched on reset. EA should be strapped to VCC for internal program executions.

This pin also receives the 12-volt programming enable voltage (VPP) during Flash programming.

XTAL1: Input to the inverting oscillator amplifier and input to the internal clock operating circuit.

XTAL2: Output from the inverting oscillator amplifier.

\section{H. Display Unit}

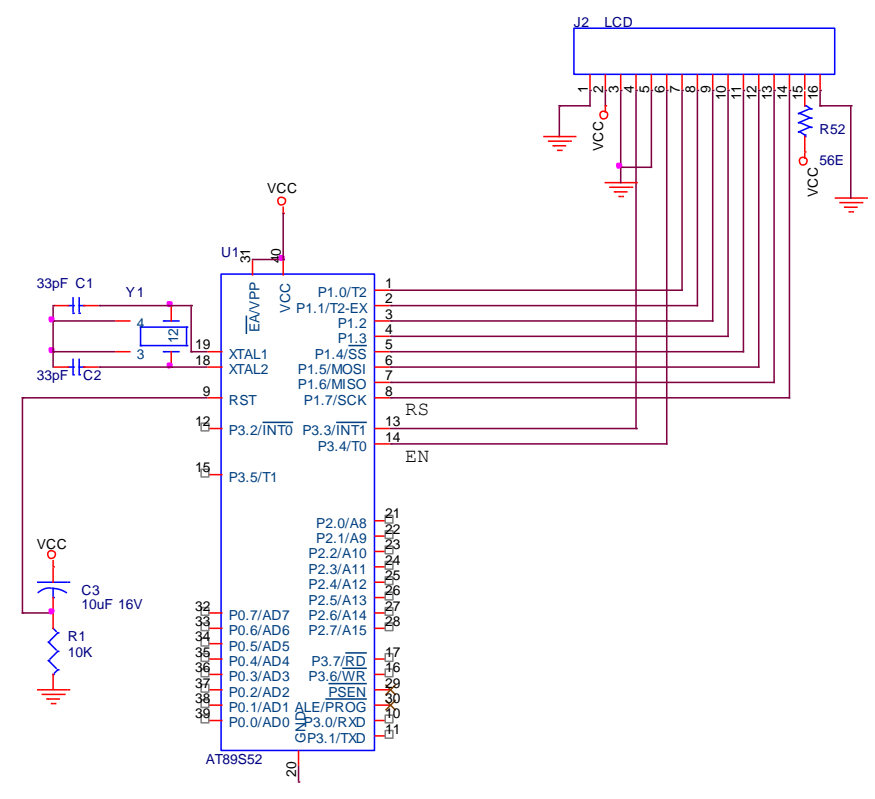

Fig 3-Display Unit

Liquid crystal displays (LCD) is an alphanumeric display and widely used in recent years as compared to LEDs. This is due to the declining prices of LCD, the ability to display numbers, characters and graphics, incorporation of a refreshing controller into the LCD, their by relieving the CPU of the task of refreshing the LCD and also the ease of programming for characters and graphics. We have used JHD162A advanced version of HD44780 based LCDs.

\section{What Can Be Automated}

Virtually anything in the home/office that is powered by electricity can be automated and/or controlled. We can control our electrical devices. We can turn our porch lights on 
automatically(Fig 4) at dark or when someone approaches and can see
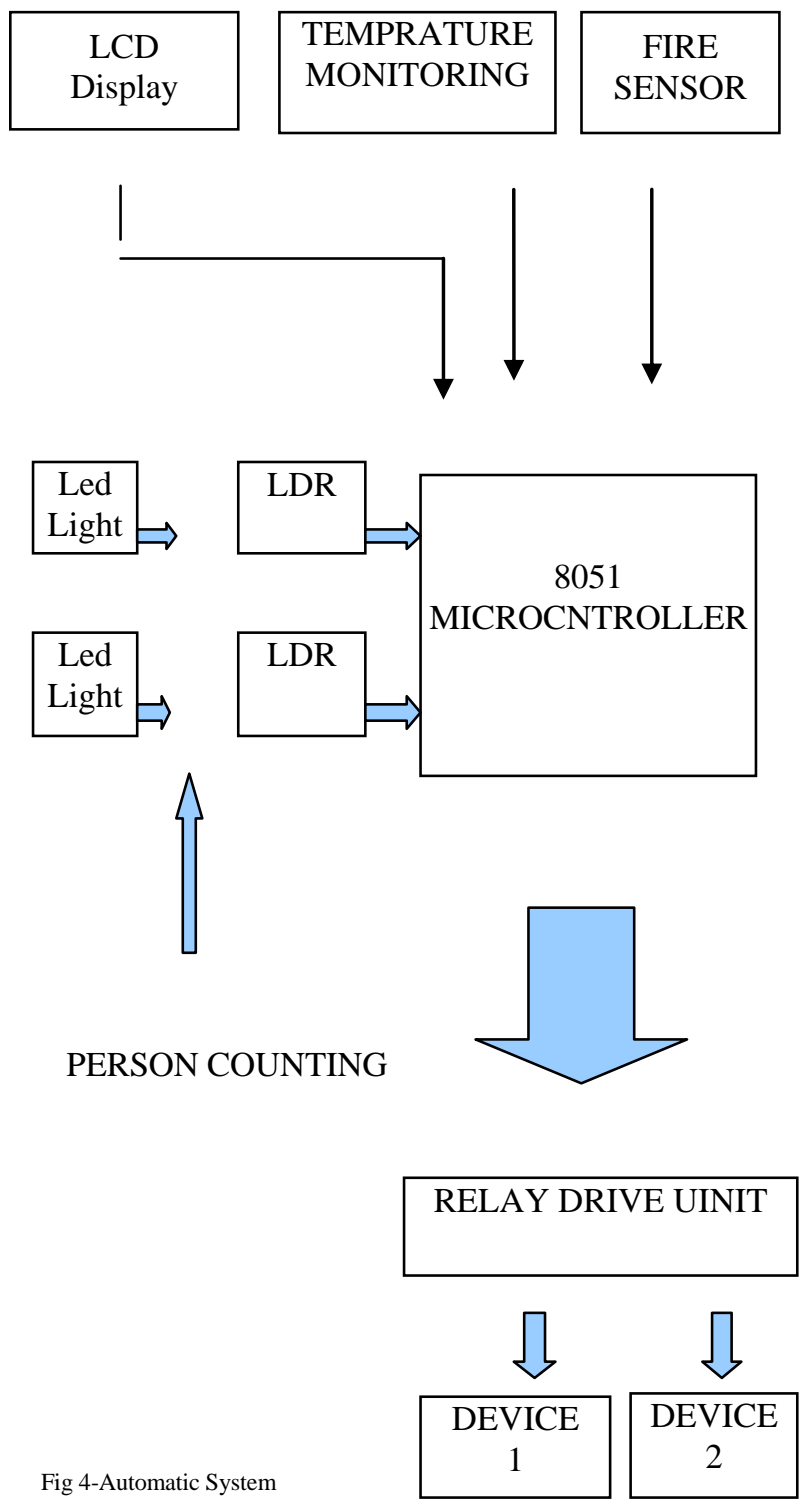

who is at the front door from any nearby television, and talk to them or unlock the door from any nearby telephone. Have the security system turn off lights, close drapes and setback the temperature when we leave and turn on the alarm system. The possibilities are only limited by our imagination.

\section{FEATURES}

\section{A. Password Based Locking System}

In this system we have ensured a safe locking system. On seeing from outside the lock would not be visible but this inbuilt locking system ensures security. This lock can be opened and closed with the help of a password which we will give using a keypad. The door will only open or close only if the password is correct else it will remain in its original state. The lock cannot be broken because to the person standing outside can just see the closed door and not the lock as it is inbuilt. The password is given with the help of controller and can be changed by simply making a small change in the program and then burning the program in the controller.

\section{B. Counter dependent automatic switching system of room}

After opening the lock when the person enters the room the counter gets incremented. Now if it is a day then the lights would not be switched on but if it is dark then the lights will automatically switch on. Now whatever may be the number of people entering the room the counter will automatically get incremented by itself and on leaving the room the counter will get decremented but the system will keep on working. Once the counter is zero in other words once everyone leaves the room the switching system will automatically stop working.

\section{Temperature controlled cooling system}

Once the person has entered the room he would not require to switch on anything everything will just happen automatically. Like if the temperature is high then the fan will switch on, on its own. Else it will remain in off state. This temperature is predefined by us in the controller. But this system will only work if there is a person in the room in other words if the counter is not zero.

\section{Light saving system}

This light saving system is used in two places for internal section and external section. If a person is not at home or sitting inside the room and it is dark outside then the lights will automatically get switched on and when its day the light will get switched off. This ensures power saving.

\section{E. $\quad$ Fire and Smoke sensor}

This part detects any fire or smoke from a fire and set an alarm or an indication.

\section{CONCLUSION}

An automated home can be a very simple grouping of controls, or it can be heavily automated where any appliance that is plugged into electrical power is remotely controlled. Costs mainly include equipment, components, furniture, and custom installation.

Ongoing costs include electricity to run the control systems, maintenance costs for the control and networking systems, including troubleshooting, and eventual cost of upgrading as standards change. Increased complexity may also increase maintenance costs for networked devices.

Learning to use a complex system effectively may take significant time and training.

Control system security may be difficult and costly to maintain, especially if the control system extends beyond the home, for instance by wireless or by connection to the internet or other networks. 
Future of Automation: Future will be of Automation of all products. Each and every product will be smart devices that we use daily and that will be controlled through a smart chip called microcontrollers. Each and Every home appliances will be controlled either by PC or hand held devices like PDA or mobile handsets. Some examples of it are when you want you can switch on/off Fan of your home by mobile handset or PC.

Smart Grid: Home automation technologies are viewed as integral additions to the Smart grid. The ability to control lighting, appliances, HVAC as well as Smart applications (load shedding, demand response, real-time power usage and price reporting) will become vital as Smart Grid initiatives are rolled out.

\section{REFERENCES}

[1] http://www.smartcomputing.com/editorial/article.asp?article=articles $\% 2$ F1995\%2Fmar95\%2Fpcn0323\%2Fpcn0323.asp retrieved 20100902

[2] "U.S. Patent 613809: Method of and apparatus for controlling mechanism of moving vessels and vehicles". United States Patent and Trademark Office. 1898-11-08. Retrieved 2010-06-16.

[3] William C. Mann (ed.) Smart technology for aging, disability and independence : the state of the science, John Wiley and Sons, 20050 471-69694-3, pp. 34-66
[4] http://www.drdobbs.com/184404040;jsessionid=IM5NJPJYWXAOFQE 1GHPCKH4ATMY32JVN Dag Spicer, If You Can't Stand the Coding, Stay Out of the Kitchen, Dr. Dobb's Journal, August 2000 , retrieved 2010 Sept 2

[5] "Home automation costs". Totalavcontrol.co.uk. Retrieved 2010-02-18.

[6] "About Us". InsteonSmartGrid.com. Retrieved 2009-11-20.

[7] Worlds Open Protocol ISO/IEC 14543-3 KNX www.knx.org

\section{AUTHORS PROFILE}

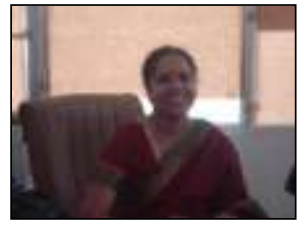

Inderpreet Kaur received the Master of Engineering from PEC University of Technology (Formerly Punjab Engineering College), Chandigarh. She is pursuing her $\mathrm{Ph} . \mathrm{D}$ in Electronics and Communication Engineering. Her area of research is optical fiber communication. She has total of 14 years of experience. She is life member of ISTE, OSI, IEI. She is in the committee of Reviewer in national and international Journals. She regularly contributes in various Journals, Magazines, and Conferences. She can be contacted at inder_preet74@yahoo.com 